\title{
New Surgical Strategy for Abdominal Wall Defects. A Successful Swine Model Experiment
}

\author{
Florina Popa* \\ Department of General Surgery, University of Medicine and Pharmacy "Iuliu Hatieganu”, Cluj-Napoca, Romania
}

Received: November 30, 2017; Published: December 06, 2017

*Corresponding author: Florina POPA, Department of General Surgery, University of Medicine and Pharmacy “Iuliu Hatieganu”Cluj-Napoca, Romania, Tel: 40755959363; Email: florinapopa11@yahoo.com

\begin{abstract}
Background: The purpose of this study was to develop a large incisional hernia pattern made on an animal that is clinically relevant, to design a new original surgical technique to repair abdominal wall defect, and to evaluate its effectiveness, aiming to apply it on patients.

Methods: Physical examination and ultrasound scan was performed at fixed intervals at 3, 7, 14 and 30 days. Postoperative complications were followed. To evaluate the resistance of the repair, the swine model went through gestation and given birth process.

Results: There are the following situations: wound infection, enteric fistula, stoma, or retraction of the abdominal wall, when direct synthetic mesh repair is inadequate. Biological meshes may be placed to achieve abdominal wall reconstruction, but they are associated with a high recurrence risk. The good result is based on the flap properties of biocompatibility and incorporation into the surrounding tissue. If some surgeons questioned the flap repair, which would not provide the required resistance, the reinforcement with the mesh would ensure it. The advantage of using the flap directly into the defect reduces the risk of infection. Mechanical test of abdominal rectus muscle across the repair site was performed and no test is better than the gestation and given birth process.

Conclusion: The technique accomplishes the reconstruction of a functional dynamic abdominal wall via the antilogous vascularized tissue flap and the mesh insertion, which will reinforce the repair. We recommend it due to the fast tissue regeneration and resistance to pressure forces on different physiological acts.
\end{abstract}

Keywords: Flap; Reconstruction; Abdominal Defect

\section{Introduction}

Despite the frequency of ventral hernia repair, there is little consensus in the literature about the ideal approach for this difficult problem. In the repair of abdominal wall defects, the surgeon must consider a multitude of factors to identify the appropriate surgical technique to accomplish the reconstructive goals [1]. The reconstruction of the abdominal wall has become a new surgical superspeciality, which needs to be investigated. Plastic surgeons use native abdominal wall whenever possible and general surgeons suggests the use of synthetic or biologic mesh in ventral hernia repairs. One particular group of patients are those with contaminated wounds, enterocutaneous fistulas, recurrent incisional hernias, where synthetic mesh is to be avoided if possible. Due to the high risk of mesh infection or mesh rejection that could lead to dramatic consequences [2]. Most recently, biologic mesh has become the new standard in high-risk patients with contaminated and dirty-infected wounds [3].
The cost of the biomaterials should not be an important factor in these cases, but this issue of price cannot be avoided, especially when hospitals do not have enough funds (in poor or developing countries) or have a limited number of meshes (in some developed countries) or, even worse, the patient must support all the costs by himself. Furthermore, this product is usually ordered on a caseby-case basis because of the expense of having large numbers $o$ matrix sheets available for intermittent use [4]. Therefore, flap surgery is the option for such cases. Flap reconstruction offers immediate and definitive wound closure, mitigating the local milieu inflammatory response and local tissue injury [5]. Although these defects can be attributed to a myriad of etiologic factors, the objectives in abdominal wall reconstruction are consistent and include the restoration of abdominal wall integrity, protection of intra-abdominal viscera, and the prevention of herniation [6]. A new experimental surgical repair, an original method for the 
reconstruction of the abdominal wall, using deep inferior epigastria artery perforator propeller flap reinforced by an on lay prosthetic mesh, is presented for the first time in this article.

\section{Methods}

\section{Animals}

An experimental protocol was developed on a swine as a model. Anesthesia was performed with atropine sulfate $0,04 \mathrm{mg} / \mathrm{kg}$ $\mathrm{SC}$, azaperone $2 \mathrm{mg} / \mathrm{kg}$ IM (Stressing, Belgium), diazepam 0,1mg/ $\mathrm{kg}$ IM, ketamine $10 \mathrm{mg} / \mathrm{kg}$ IM (Vetased, Romania). This protocol of sedation allowed safe transportation to the preparation room. Protocol was administered to have effect on the induction of general anesthesia (1-2mg/kg IV) (Norfolk, Northern Ireland). The swine was incubated with a straight laryngoscope blade using a number 5.5 - 6 end tracheal tube. To maintain anesthesia, isoflurane was delivered in $100 \%$ oxygen (Dragger Fabius Plus XL, Dragger Medical AG \& Co - Germany). ECG, respiratory rate, oxygen saturation, pulse, esophageal temperature, ETCO2, FiO2 and anesthetic gas concentration were monitored throughout the surgical procedure. Postoperative analgesia - meloxicam $0.4 \mathrm{mg} /$ $\mathrm{kg}$ IM/24 h, Lexicon (Nor brook Laboratories Limited, Northern Ireland) was also performed.

\section{Abdominal Wall Defect Creation}

The swine model underwent survival surgery which consisted in creating a standard ellipse $5 \mathrm{~cm}$ in width and $10 \mathrm{~cm}$ in length abdominal wall defect, using a surgical steel blade no. 23, through the fascia of the midline linea alba. The peritoneum immediately below the facial incision was kept intact. Full-thickness skin flap$1.5 \mathrm{~cm}$ lateral to the midline - was raised through the vascular prefascial plane. The 1:2 ratio of the flap length to width was maintained to prevent flap ischemia.

\section{New Surgical Repair}

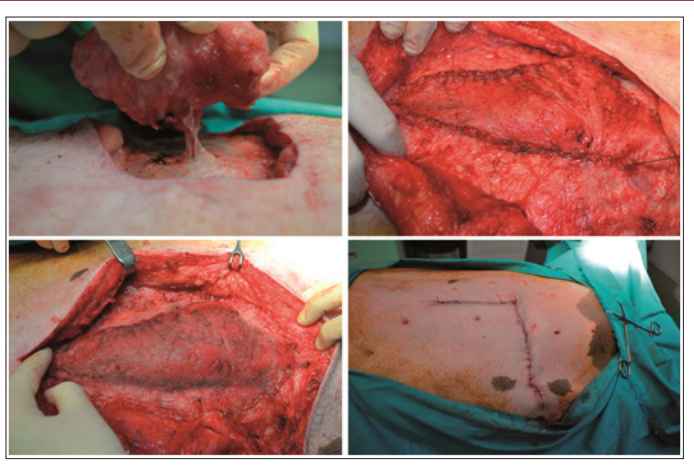

Figure 1: The new technique: The flap is harvest based on a single perforator; fixing the flap into the defect; prosthetic mesh reinforcement over the flap repair; skin closer with intra-dermal suture.

Simultaneously, the repair was done using a de-epithelialized flap, harvested in a supine position. An elliptic transverse skin paddle was drawn with a surgical marker on the inferior hemiabdomen. The flap was designed as an island flap based on a single perforator, in our case DIEP, and was rotated through 90 degrees to cover the defect. The flap was then inset without tension and the donor site area was closed first. After the flap was fixed into the defect, the repair was supplemented with a lightweight polypropylene-mesh, in the onlay position, extended beyond the line of the closure by $3 \mathrm{~cm}$ in all directions. The mesh was fixed with a continuous suture around the periphery, using a heavy gauge non-absorbable suture. The fascia closure was tension-free, using a continuous suture and the skin was closed with intra-dermal suture using Monocryl 3.0 (Figure 1). To determine the repair strength, due to lack of an tensile tester or other equipped device, I decided to have a tensile testing of the swine model by choosing a natural way. Because a month after surgery the swine model underwent the reproduction process.

\section{Results}

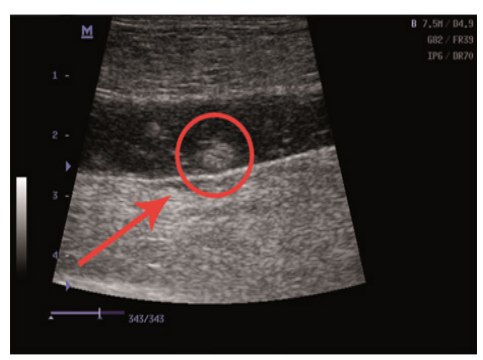

Figure 2: Ultrasound scan identified small hematomas formations.

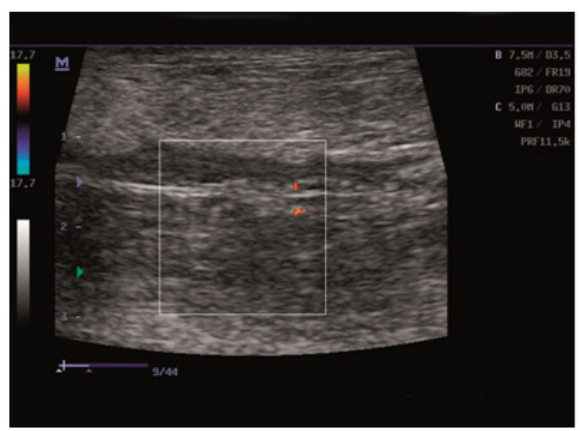

Figure 3: Ultrasound scans: normal vessel flow.

Physical examination and ultrasound scan were performed at fixed intervals at $3,7,14$ and 30 days. Then complications followed: infection, seroma/hematoma, extrusion, hernia recurrence, dehiscence, and necrosis. After a three-day-evaluation a slight edema can be noticed during the physical examination. There are no other concerns regarding the wound healing. Ultrasound scan identified small hematomas formations above the repair, in the subfascial space, even if the mesh was well positioned (Figure 2). Examining the flap perfusion, the perforator vessel was identified and the vessel flow was normal (Figure 3). Seven days later after the surgery, the flap seems to be well incorporated into repair, excepting the extremities of the defect, where mesh elevation is detected. Clinical examination shows a normal process of the wound healing (Figure 4). No changes occur at the two-week evaluation. After a month the results reveal the good repair of the abdominal wall without complications at the US evaluation and also with optimal cosmetic results (Figure 5). After the breeding, the normal gestation period was carried on without any problems, and 
the swine model gave birth to four piglets. Both mother and piglets are healthy. One-week physical examination of the abdominal wall swine model shows no recurrent hernia being present (Figure 6).

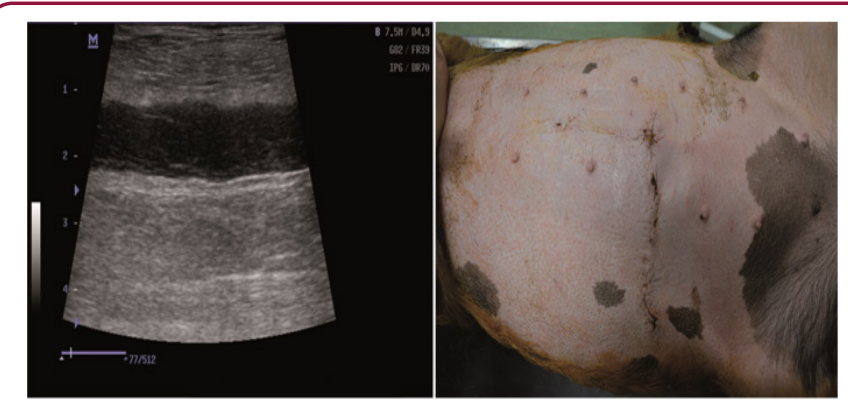

Figure 4: Normal process of wound healing: Ultrasound scan/Clinical examination.

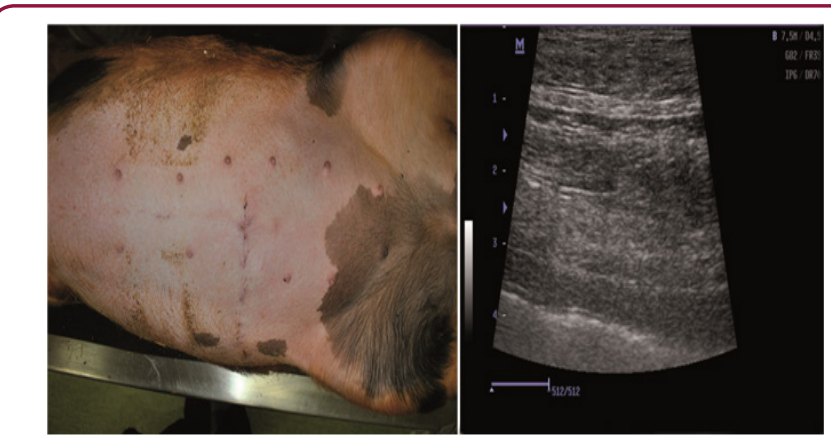

Figure 5: One moth physical evaluation/ ultrasound image.

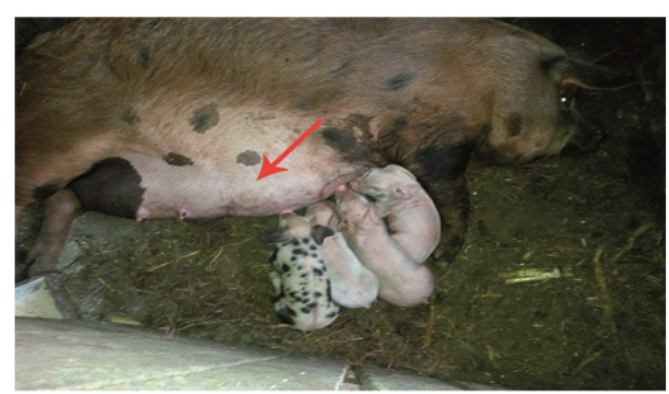

Figure 6: One week after the birth.

\section{Discussion}

Several options exist for the reconstruction of the abdominal wall defects. The idea for this original repair started with the question: why a perfect mesh and not a combination using the original material reinforced with a mesh? There are many different types of meshes available, and even more products are being brought to the marketplace, having a commercial interest. It is difficult to browse through all of these new products, without longterm clinical and experimental data to support their use [4]. If we add the high costs in purchasing these meshes, all of these hamper and delay the repair process. There are situations, such as wound infection, enteric fistula, stoma, or retraction of the abdominal wall, when direct mesh repair is inadequate. Biological meshes may be placed to achieve abdominal wall reconstruction, but they are associated with a high recurrence risk because they weaken as they dissolve [2]. Placing the flap into the defect, prevents the development of complications, which can jeopardize the repair. The only technique is popular among surgeons because it avoids direct contact with bowel and imparts less tension on the repair [7]. Mechanical test of abdominal rectus muscle across the repair site was performed and no test is better than the gestation and given birth process. Results show that the repaired area did not record any changes throughout all the process and it proved to be a resistant repair.

Among the postoperative complications, only hematomas were recorded, and they were resolved by natural drainage. Hematomas are susceptible of infection and can easily provide the medium for bacterial overgrowth. Any evidence of infection with hematoma may require evacuation of the hematoma to prevent removal of the mesh. No infection, no superficial or deep wound dehiscence, no recurrence even after the birth process was recorded in the swine model. Mesh infection is given by local or systemic factors. Additionally, the properties of the mesh material affect the degree of local inflammatory response and fibro vascular tissue incorporation [4]. The good result is based on the flap properties of biocompatibility and incorporation into the surrounding tissue. And, if some surgeons questioned whether the flap repair would provide the required resistance, the reinforcement with the mesh will ensure it. This technique allows the preservation of the perforator vessel, reducing the risk of subsequent skin necrosis. From an esthetic point of view the repair, combining the two methods used on the swine model, had an excellent result, which did not undergo any changes during the gestation process. The advantage of using the flap directly into the defect reduces the risk of infection. The recurrence rate is an important element to establish the efficiency of surgical treatment [2]. Long-term data analysis, with large case studies, is still needed to obtain detailed information about recurrence, particularly in the assessment of new techniques.

\section{Conclusion}

The repair accomplishes the reconstruction of a functional dynamic abdominal wall via the antilogous vascularized tissue flap. Mesh reinforcement of the repair provides resistance under constant pressure and can be indicated to treat young patients. Long-term clinical and experimental data are needed to support its use.

\section{References}

1. Petro CC, Rosen MJ (2014) Repair of ventral abdominal wall hernias. Gastrointestinal Tract and abdomen.

2. Crovella F, Bartone G, Fei L (2008) Incisional Hernia. Springer-Verlag Milan, Italia.

3. Rifat Latifi (2016) Practical Approaches to Definitive Reconstruction of Complex Abdominal Wall Defects. World Journal of Surgery 40(4): 836848.

4. Grevious MA, Cohen M, Jean-Pierre F, Herrmann GE (2006) The use of prosthetics in abdominal wall reconstruction. Clin Plast Surg 33(2): 181197.

5. Baumann DP, Butler CE (2013) Soft tissue coverage in abdominal wall reconstruction. Surg Clin North Am 93(5): 1199-1209. 
6. Justin M, Sacks, Justin M. Broyles, Donald P. Baumann (2012) Flap Coverage of Anterior Abdominal Wall Defects. Semin Plast Surg 26(1): 36-39.
7. Shell DH 4th, de la Torre J, Andrades P, Vasconez LO (2008) Open repair of ventral incisional hernias. Surg Clin North Am 88(1): 61-83.

Assets of Publishing with us
BIOMEDICAL

